\title{
Convergence Rates of Neumann problems for Stokes Systems
}

\author{
Shu $\mathrm{Gu}^{*}$
}

\begin{abstract}
In quantitative homogenization of the Neumann problems for Stokes systems with rapidly oscillating periodic coefficients, this paper studies the convergence rates of the velocity in $L^{2}$ and $H^{1}$ as well as those of the pressure term in $L^{2}$, without any smoothness assumptions on the coefficients.
\end{abstract}

Keywords. Convergence rates; Stokes systems; Homogenization; Neumann problems.

\section{Introduction and Main Results}

In this paper, we would like to investigate the convergence rates of Neumann problems for Stokes systems with rapidly oscillating periodic coefficients. Specifically, we'd like to consider the following Neumann problem for Stokes system in a bounded domain $\Omega \subset \mathbb{R}^{d}$,

$$
\left\{\begin{aligned}
\mathcal{L}_{\varepsilon}\left(u_{\varepsilon}\right)+\nabla p_{\varepsilon} & =F & & \text { in } \Omega, \\
\operatorname{div}\left(u_{\varepsilon}\right) & =g & & \text { in } \Omega, \\
\frac{\partial u_{\varepsilon}}{\partial \nu_{\varepsilon}}-p_{\varepsilon} n & =f & & \text { on } \partial \Omega,
\end{aligned}\right.
$$

where $n$ denotes the outward unit normal to $\partial \Omega$. Throughout this paper, we use the summation convention and let $\varepsilon>0$ be a small parameter. We define the second-order elliptic operator in divergence form $\mathcal{L}_{\varepsilon}$ associated with coefficient matrix $A$ by

$$
\mathcal{L}_{\varepsilon}=-\operatorname{div}(A(x / \varepsilon) \nabla)=-\frac{\partial}{\partial x_{i}}\left[a_{i j}^{\alpha \beta}\left(\frac{x}{\varepsilon}\right) \frac{\partial}{\partial x_{j}}\right]
$$

where $1 \leq i, j, \alpha, \beta \leq d$, and the conormal derivative of system (1.1) on $\partial \Omega$ is defined by

$$
\left(\frac{\partial u_{\varepsilon}}{\partial \nu_{\varepsilon}}\right)^{\alpha}-p_{\varepsilon} n_{\alpha}=n_{i}(x) a_{i j}^{\alpha \beta}(x / \varepsilon) \frac{\partial u_{\varepsilon}^{\beta}}{\partial x_{j}}-p_{\varepsilon}(x) n_{\alpha}(x) .
$$

${ }^{*}$ Supported in part by NSF grant DMS-1161154 
We assume that the coefficient matrix $A(y)=\left(a_{i j}^{\alpha \beta}(y)\right)$ is real, bounded measurable, and it satisfies the ellipticity condition:

$$
\mu|\xi|^{2} \leq a_{i j}^{\alpha \beta}(y) \xi_{i}^{\alpha} \xi_{j}^{\beta} \leq \frac{1}{\mu}|\xi|^{2} \quad \text { for } y \in \mathbb{R}^{d} \text { and } \xi=\left(\xi_{i}^{\alpha}\right) \in \mathbb{R}^{d \times d}
$$

where $\mu>0$, and also the periodicity condition,

$$
A(y+z)=A(y) \quad \text { for } y \in \mathbb{R}^{d} \text { and } z \in \mathbb{Z}^{d} .
$$

A function satisfying (1.5) will be called 1-periodic.

The homogenization theory of Neumann problems for Stokes systems tells us that, $u_{\varepsilon}-f_{\Omega} u_{\varepsilon}$ converges to $u_{0}-f_{\Omega} u_{0}$ weakly in $H^{1}$, and $p_{\varepsilon}-f_{\Omega} p_{\varepsilon}$ converges to $p_{0}-f_{\Omega} p_{0}$ weakly in $L^{2}$, given suitable $F, f$ and $g$. Here $\left(u_{0}, p_{0}\right) \in H^{1}\left(\Omega ; \mathbb{R}^{d}\right) \times L^{2}(\Omega)$ is the weak solution of the associated homogenized problem with constant coefficients,

$$
\left\{\begin{aligned}
\mathcal{L}_{0}\left(u_{0}\right)+\nabla p_{0} & =F & & \text { in } \Omega \\
\operatorname{div}\left(u_{0}\right) & =g & & \text { in } \Omega \\
\frac{\partial u_{0}}{\partial \nu_{0}}-p_{0} n & =f & & \text { on } \partial \Omega .
\end{aligned}\right.
$$

The nature and primary question will be how fast does it converge. Our main purpose is to study the optimal convergence rate of $\left\|u_{\varepsilon}-u_{0}\right\|_{L^{2}(\Omega)}$, as $\varepsilon \rightarrow 0$. The result is given in the following theorem.

Theorem 1.1. Let $\Omega$ be a bounded $C^{1,1}$ domain. Suppose A satisfies ellipticity condition (1.4) and periodicity condition (1.5). Given $F \in L^{2}\left(\Omega ; \mathbb{R}^{d}\right)$ and $f \in H^{1 / 2}\left(\partial \Omega ; \mathbb{R}^{d}\right)$ satisfying the compatibility condition

$$
\int_{\Omega} F+\int_{\partial \Omega} f=0
$$

for any $g \in H^{1}(\Omega)$, let $\left(u_{\varepsilon}, p_{\varepsilon}\right),\left(u_{0}, p_{0}\right)$ be the weak solutions of Neumann problems (1.1), (1.6), respectively. If $\int_{\Omega} u_{\varepsilon}=\int_{\Omega} u_{0}=0$, then

$$
\left\|u_{\varepsilon}-u_{0}\right\|_{L^{2}(\Omega)} \leq C \varepsilon\left\|u_{0}\right\|_{H^{2}(\Omega)}
$$

where the constant $C$ depends only on $\mu, d$, and $\Omega$.

Theorem 1.1 gives us the order $O(\varepsilon)$ convergence of the velocity in $L^{2}$, which is optimal in the sense of $\left\|u_{0}\right\|_{H^{2}(\Omega)}$. The other important result of this paper, which is shown in the next theorem, is that the two-scale expansion of $\left(u_{\varepsilon}, p_{\varepsilon}\right)$ has optimal $O\left(\varepsilon^{1 / 2}\right)$ rates in $H^{1} \times L^{2}$.

For simplicity, we will use the notation $h^{\varepsilon}(x)=h(x / \varepsilon)$, for any function $h$. Here $(\chi, \pi)$ are the correctors associated with $A$, defined as in (2.5), and $S_{\varepsilon}$ is the Steklov smoothing operator introduced in (2.12). 
Theorem 1.2. Let $\Omega$ be a bounded $C^{1,1}$ domain. Suppose A satisfies ellipticity condition (1.4) and periodicity condition (1.5). Let $\left(u_{\varepsilon}, p_{\varepsilon}\right)$ and $\left(u_{0}, p_{0}\right)$ be the same as in Theorem 1.1. If $\int_{\Omega} u_{\varepsilon}=\int_{\Omega} u_{0}=0$, then

$$
\left\|u_{\varepsilon}-u_{0}-\varepsilon \chi^{\varepsilon} S_{\varepsilon}\left(\nabla \widetilde{u}_{0}\right)\right\|_{H^{1}(\Omega)} \leq C \sqrt{\varepsilon}\left\|u_{0}\right\|_{H^{2}(\Omega)},
$$

where $\widetilde{u}_{0}$ is the extension of $u_{0}$ defined as in (3.1). Moreover, if $\int_{\Omega} p_{\varepsilon}=\int_{\Omega} p_{0}=0$, then

$$
\left\|p_{\varepsilon}-p_{0}-\left[\pi^{\varepsilon} S_{\varepsilon}\left(\nabla \widetilde{u}_{0}\right)-f_{\Omega} \pi^{\varepsilon} S_{\varepsilon}\left(\nabla \widetilde{u}_{0}\right)\right]\right\|_{L^{2}(\Omega)} \leq C \sqrt{\varepsilon}\left\|u_{0}\right\|_{H^{2}(\Omega)}
$$

The constants $C$ in (1.9) and (1.10) depend only on $\mu, d$, and $\Omega$.

There are relatively fewer known $L^{2}$ convergence rates results for Neumann problems than for Dirichlet cases. For the scalar elliptic equation $\mathcal{L}_{\varepsilon}\left(u_{\varepsilon}\right)=-\operatorname{div}\left(A(x / \varepsilon) \nabla u_{\varepsilon}\right)=F$ in $\Omega$ with Neumann condition $\frac{\partial u_{\varepsilon}}{\partial \nu_{\varepsilon}}=0$ on $\partial \Omega$, the estimate $\left\|u_{\varepsilon}-u_{0}\right\|_{L^{2}(\Omega)} \leq C \varepsilon\|F\|_{H^{2}(\Omega)}$ was proved by Griso [7] for $C^{1,1}$ domains with bounded measurable coefficients, by using the method of periodic unfolding (see [2,3]). The same result was also proved by Moskow and Vogelius [17] for curvilinear convex polygons $\Omega$ in $\mathbb{R}^{2}$.

For the system case, consider the standard second-order elliptic systems $\mathcal{L}_{\varepsilon}\left(u_{\varepsilon}\right)=F$ in $\Omega$ with Neumann condition $\frac{\partial u_{\varepsilon}}{\partial \nu_{\varepsilon}}=g$ on $\partial \Omega$, Kenig, Lin and Shen [12] have shown that the better estimate (1.8) holds in bounded Lipschitz domain $\Omega$, under additional assumption that $A$ is Hölder continuous. Let

$$
v_{\varepsilon}=u_{\varepsilon}-u_{0}-\varepsilon \chi^{\varepsilon} \nabla u_{0}
$$

be the difference between $u_{\varepsilon}$ and its first order approximation. The approach used in [12] was based on the explicit computation of conormal derivative of $v_{\varepsilon}$ and uniform regularity estimates for the $L^{2}$ Neumann problem derived in [14,15]. Moreover, if additionally assume that $A$ is Hölder continuous and symmetric, it was proved in [12] that $\left\|v_{\varepsilon}\right\|_{H^{1 / 2}(\partial \Omega)} \leq$ $C \varepsilon\left\|u_{0}\right\|_{H^{2}(\Omega)}$.

Nevertheless, the method used in [12] cannot be applied to operators with bounded measurable coefficients, since the correctors $\chi$ are not necessarily bounded. With the help of the Steklov smoothing operator, Suslina [24, 25] was able to establish the $O(\varepsilon)$ estimate (1.8) in $L^{2}$ for a broader class of elliptic operators, which includes the standard elliptic systems in divergence form. Instead, $u_{0}+\varepsilon \chi^{\varepsilon} S_{\varepsilon}\left(\nabla \widetilde{u}_{0}\right)$ was used as the first order approximation of $u_{\varepsilon}$, and the following difference

$$
v_{\varepsilon}=u_{\varepsilon}-u_{0}-\varepsilon \chi^{\varepsilon} S_{\varepsilon}\left(\nabla \widetilde{u}_{0}\right)
$$

was adopted, where $S_{\varepsilon}$ is the Steklov smoothing operator and $\widetilde{u}_{0}$ is an extension of $u_{0}$ to $\mathbb{R}^{d}$. For elliptic Neumann problems, the $O(\sqrt{\varepsilon})$ convergence (1.9) in $H^{1}$ was obtained by the estimate of the boundary layer corrector term and the following sharp convergence rates for homogenization in the whole space $\left\|u_{\varepsilon}-u_{0}\right\|_{L^{2}\left(\mathbb{R}^{d}\right)} \leq C \varepsilon\|F\|_{L^{2}\left(\mathbb{R}^{d}\right)}$. The $O(\varepsilon)$ estimate (1.8) in $L^{2}$ then can be deduced by applying the estimate (1.9) to adjoint problems and a duality argument. 
However, the case of Stokes systems certainly does not fit the standard framework of standard second-order elliptic systems in divergence form. As expected, in the study of Stokes or Navier-Stokes systems, the main difficulty is to deal with the pressure term $p_{\varepsilon}$. Because the conormal derivative includes the pressure term, it's not appropriate to use boundary layer corrector term as Suslina did for the elliptic Neumann problems [25] or as in [8] for Dirichlet problems of Stokes systems. Instead, in this paper we use a more direct approach which helps us to avoid the convergence rates result for the whole space. The key intermediate step we use is the following,

$$
\left|\int_{\Omega} A^{\varepsilon} \nabla v_{\varepsilon} \cdot \nabla \varphi\right| \leq C\left\|u_{0}\right\|_{H^{2}(\Omega)}\left[\|\operatorname{div}(\varphi)\|_{L^{2}(\Omega)}+\varepsilon^{1 / 2}\|\nabla \varphi\|_{L^{2}\left(\Omega_{2 \varepsilon}\right)}+\varepsilon\|\nabla \varphi\|_{L^{2}(\Omega)}\right],
$$

for any $\varphi \in H^{1}\left(\Omega ; \mathbb{R}^{d}\right)$, and $\Omega_{\varepsilon}=\{x \in \Omega$ : $\operatorname{dist}(x, \partial \Omega)<\varepsilon\}$. By choosing the suitable test function in the above key step, and provided the estimates of the correctors $(\chi, \pi)$ and their duals $(\Phi, q)$, we are able to establish the $O(\sqrt{\varepsilon})$ error estimates for the twoscale expansions of $\left(u_{\varepsilon}, p_{\varepsilon}\right)$ in $H^{1} \times L^{2}$, which is stated in Theorem 1.2. We emphasize that convergence rates of the pressure term in $L^{2}$ require an explicit computation of the pressure term corresponding to $v_{\varepsilon}$. At last, we apply the $O(\sqrt{\varepsilon})$ estimates to adjoint problems, and further obtain the $O(\varepsilon)$ estimate in $L^{2}$ through duality argument as well as the key intermediate step.

The theory of homogenization for operators with rapidly oscillating coefficients has been playing a vital part in describing the behavior of composite materials, which contain two or more finely mixed constituents. For Stokes systems with rapidly oscillating coefficients, one application would be studying the groundwater behavior in layered aquifer structures. Another application is related to the incompressible free fluid in porous media. If the ratio of the size of the porous to the period is $O(\varepsilon)$, and consider a viscosity function which characterizes different viscosities in fluid and solid parts, then the homogenization theory in porous media and the derivation of Darcy's law may be regarded as one type of homogenization of Stokes systems with rapidly oscillating periodic coefficients.

The problem of convergence rates has been playing an essential role in quantitative homogenization. Most recent work on the problem of convergence rates in periodic homogenization may be found in [4, 6- 8, 11-13, 18, 20, 25, 27] and their references.

We now mention the potential applications of these results. Inspired by recent paper of Shen [22] on systems of linear elasticity, we expect to establish the boundary Lipschitz estimates in $C^{1, \alpha}$ domains for Neumann problems of Stokes systems with rapidly oscillating periodic coefficients, using convergence rates in $H^{1}$ and $L^{2}$ rather than the compactness method introduced by Avellaneda and Lin [1]. We may also use the result to investigate the $C^{\alpha}, W^{1, p}$, and $L^{p}$ estimates in $C^{1}$ domains with VMO or Hölder continuous coefficients.

The paper is organized as follows. In Section 2 we state the homogenization theory of Stokes systems, estimates of dual correctors $(\Phi, q)$ and also the Steklov smoothing operator. In Section 3 we derive the key intermediate step by explicit computation of the system and conormal derivative that $v_{\varepsilon}$ satisfies, and further we prove the $O(\sqrt{\varepsilon})$ rate of $u_{\varepsilon}$ in $H^{1}$. In Section 4 we need a more explicit computation to prove the $O(\sqrt{\varepsilon})$ for 
the two-scale expansion of the pressure term $p_{\varepsilon}$. Finally, our main theorem Theorem 1.1 is proved in Section 5 by applying Theorem 1.2 to adjoint systems and also a duality argument.

Throughout this paper, we use $Y=[0,1)^{d}$ to denote the unit cube and define the $L^{1}$ average of $f$ over the set $E$ as

$$
f_{E} f=\frac{1}{|E|} \int_{E} f
$$

We will use $C$ to denote constants that may depend on $d, \mu$ or $\Omega$, but never on $\varepsilon$.

\section{Preliminaries}

\subsection{Weak solution of Stokes systems}

We use this subsection to review the weak solutions of Stokes systems and the qualitative homogenization theorem for Neumann problems. Details may be found in [9, 10, 16].

Let $\Omega$ be a bounded Lipschitz domain in $\mathbb{R}^{d}$. Given $F \in H^{-1}\left(\Omega ; \mathbb{R}^{d}\right)$ and $g \in L^{2}(\Omega)$, consider the following Stokes system

$$
\left\{\begin{aligned}
\mathcal{L}_{\varepsilon}\left(u_{\varepsilon}\right)+\nabla p_{\varepsilon} & =F \\
\operatorname{div}\left(u_{\varepsilon}\right) & =g
\end{aligned} \quad \text { in } \quad \Omega\right.
$$

We define the bilinear form $a_{\varepsilon}(\cdot, \cdot)$ by

$$
a_{\varepsilon}(u, v)=\int_{\Omega} a_{i j}^{\alpha \beta}\left(\frac{x}{\varepsilon}\right) \frac{\partial u^{\beta}}{\partial x_{j}} \frac{\partial v^{\alpha}}{\partial x_{i}} d x, \quad \forall u, v \in H^{1}\left(\Omega ; \mathbb{R}^{d}\right),
$$

then we say that $\left(u_{\varepsilon}, p_{\varepsilon}\right) \in H^{1}\left(\Omega ; \mathbb{R}^{d}\right) \times L^{2}(\Omega)$ is a weak solution of system (2.1) if

$$
a_{\varepsilon}\left(u_{\varepsilon}, \varphi\right)-\int_{\Omega} p_{\varepsilon} \operatorname{div}(\varphi)=\langle F, \varphi\rangle, \quad \forall \varphi \in C_{0}^{1}\left(\Omega ; \mathbb{R}^{d}\right),
$$

and $\operatorname{div}\left(u_{\varepsilon}\right)=g$ in $\Omega$ (in the sense of distribution).

Theorem 2.1. Let $\Omega$ be a bounded Lipschitz domain in $\mathbb{R}^{d}$. Suppose $A(y)$ satisfies the ellipticity condition (1.4) and given $F \in H^{-1}\left(\Omega ; \mathbb{R}^{d}\right), g \in L^{2}(\Omega)$ and $f \in H^{-1 / 2}\left(\partial \Omega ; \mathbb{R}^{d}\right)$ satisfying the compatibility condition (1.7). Then there exist a unique (up to constants) $u_{\varepsilon} \in H^{1}\left(\Omega ; \mathbb{R}^{d}\right)$ and $p_{\varepsilon} \in L^{2}(\Omega)$ satisfying the following weak formulation

$$
a_{\varepsilon}\left(u_{\varepsilon}, \varphi\right)-\int_{\Omega} p_{\varepsilon} \operatorname{div}(\varphi) d x=\int_{\Omega} F \varphi d x+\int_{\partial \Omega} f \varphi d S, \quad \forall \varphi \in H^{1}\left(\Omega ; \mathbb{R}^{d}\right),
$$

then we say $\left(u_{\varepsilon}, p_{\varepsilon}\right)$ is a weak solution of Stokes system (2.1) with Neumann boundary condition $\frac{\partial u_{\varepsilon}}{\partial \nu_{\varepsilon}}-p_{\varepsilon} n=f$ on $\partial \Omega$. Moreover, if $\int_{\Omega} u_{\varepsilon} d x=0$, then

$$
\left\|u_{\varepsilon}\right\|_{H^{1}(\Omega)}+\left\|p_{\varepsilon}-f_{\Omega} p_{\varepsilon}\right\|_{L^{2}(\Omega)} \leq C\left\{\|F\|_{H^{-1}(\Omega)}+\|g\|_{L^{2}(\Omega)}+\|f\|_{H^{-1 / 2}(\partial \Omega)}\right\},
$$

where $C$ depends only on $d, \mu$, and $\Omega$. 
Proof. The proof is based on the Lax-Milgram Theorem. We skip the details here.

Remark 2.2. Suppose $\Omega$ is $C^{1,1}$ and $A$ is a constant matrix, and provided that $F \in$ $L^{2}\left(\Omega ; \mathbb{R}^{d}\right), g \in H^{1}(\Omega)$ and $f \in H^{1 / 2}\left(\partial \Omega ; \mathbb{R}^{d}\right)$, then the weak solution $(u, p)$ given by Theorem [2.1, is in $H^{2}\left(\Omega ; \mathbb{R}^{d}\right) \times H^{1}(\Omega)$. Moreover, if $\int_{\Omega} u d x=0$, then

$$
\|u\|_{H^{2}(\Omega)}+\|\nabla p\|_{L^{2}(\Omega)} \leq C\left\{\|F\|_{L^{2}(\Omega)}+\|g\|_{H^{1}(\Omega)}+\|f\|_{H^{1 / 2}(\partial \Omega)}\right\},
$$

where $C$ depends only on $d, \mu$, and $\Omega$ (see e.g. [5]).

\subsection{Correctors and Homogenization theorem}

For each $1 \leq j, \beta \leq d$, we define the 1 -periodic functions $\left(\chi_{j}^{\beta}, \pi_{j}^{\beta}\right) \in H_{\mathrm{loc}}^{1}\left(\mathbb{R}^{d} ; \mathbb{R}^{d}\right) \times L_{\mathrm{loc}}^{2}\left(\mathbb{R}^{d}\right)$ as the correctors for the Stokes system (2.1), which satisfy the following cell problem

$$
\left\{\begin{aligned}
& \mathcal{L}_{1}\left(\chi_{j}^{\beta}+P_{j}^{\beta}\right)+\nabla \pi_{j}^{\beta}=0 \text { in } \mathbb{R}^{d}, \\
& \operatorname{div}\left(\chi_{j}^{\beta}\right)=0 \quad \text { in } \mathbb{R}^{d}, \\
& \int_{Y} \pi_{j}^{\beta}=0, \quad \int_{Y} \chi_{j}^{\beta}=0,
\end{aligned}\right.
$$

where $P_{j}^{\beta}=P_{j}^{\beta}(y)=y_{j} e^{\beta}=y_{j}(0, \cdots, 1, \cdots, 0)$ with 1 in the $\beta^{\text {th }}$ position. Existence of such functions can be found in [8]. The homogenized system for the Stokes system (2.1) is given by

$$
\left\{\begin{aligned}
\mathcal{L}_{0}\left(u_{0}\right)+\nabla p_{0} & =F \\
\operatorname{div}\left(u_{0}\right) & =g
\end{aligned}\right.
$$

where $\mathcal{L}_{0}=-\operatorname{div}(\widehat{A} \nabla)$ is a second-order elliptic operator with constant coefficients $\widehat{A}=$ $\left(\widehat{a}_{i j}^{\alpha \beta}\right)$, with

$$
\widehat{a}_{i j}^{\alpha \beta}=\int_{Y}\left[a_{i j}^{\alpha \beta}(y)+a_{i k}^{\alpha \gamma}(y) \frac{\partial \chi_{j}^{\gamma \beta}(y)}{\partial y_{k}}\right] d y .
$$

We should remark that $(\widehat{A})^{*}=\widehat{A^{*}}$, and the effective matrix $\widehat{A}$ is also elliptic. The following is a homogenization theorem for Neumann problem for the Stokes system.

Theorem 2.3. Suppose $A(y)$ satisfies the ellipticity (1.4) and periodicity (1.5) conditions. Let $\Omega$ be a bounded Lipschitz domain. Let $\left(u_{\varepsilon}, p_{\varepsilon}\right) \in H^{1}\left(\Omega ; \mathbb{R}^{d}\right) \times L^{2}(\Omega)$ be a weak solution of Neumann problem (1.1) in the sense of (2.2), provided that $F \in H^{-1}\left(\Omega ; \mathbb{R}^{d}\right), g \in L^{2}(\Omega)$ and $f \in H^{-1 / 2}\left(\partial \Omega ; \mathbb{R}^{d}\right)$ satisfying the compatibility condition (1.7). Assume that $\int_{\Omega} u_{\varepsilon}=$ $\int_{\Omega} p_{\varepsilon}=0$, then as $\varepsilon \rightarrow 0$,

$$
\left\{\begin{aligned}
u_{\varepsilon} & \rightarrow u_{0} \quad \text { strongly in } L^{2}\left(\Omega ; \mathbb{R}^{d}\right), \\
u_{\varepsilon} & \rightarrow u_{0} \quad \text { weakly in } H^{1}\left(\Omega ; \mathbb{R}^{d}\right), \\
p_{\varepsilon} & \rightarrow p_{0} \quad \text { weakly in } L^{2}(\Omega), \\
A(x / \varepsilon) \nabla u_{\varepsilon} & \rightarrow \widehat{A} \nabla u_{0} \quad \text { weakly in } L^{2}\left(\Omega ; \mathbb{R}^{d \times d}\right) .
\end{aligned}\right.
$$


Moreover, $\int_{\Omega} u_{0}=\int_{\Omega} p_{0}=0$ and $\left(u_{0}, p_{0}\right)$ is the weak solution of the homogenized problem (1.6), in the following weak sense

$$
\int_{\Omega} \widehat{a}_{i j}^{\alpha \beta} \frac{\partial u_{0}^{\beta}}{\partial x_{j}} \frac{\partial \varphi^{\alpha}}{\partial x_{i}} d x-\int_{\Omega} p_{0} \operatorname{div}(\varphi) d x=\int_{\Omega} F \varphi d x+\int_{\partial \Omega} f \varphi d S, \quad \forall \varphi \in H^{1}\left(\Omega ; \mathbb{R}^{d}\right) .
$$

Proof. The proof of Theorem 2.3 uses Tartar's oscillating testing function method. Details can be found in [9].

\subsection{Dual correctors of Stokes systems}

This subsection is used to introduce the dual correctors $\left(\Phi_{k i j}^{\alpha \beta}, q_{i j}^{\beta}\right)$ of Stokes systems and their properties. More details can be found in [8].

For $1 \leq i, j, \alpha, \beta \leq d$, we let

$$
b_{i j}^{\alpha \beta}(y)=a_{i j}^{\alpha \beta}(y)+a_{i k}^{\alpha \gamma}(y) \frac{\partial}{\partial y_{k}}\left(\chi_{j}^{\gamma \beta}\right)-\widehat{a}_{i j}^{\alpha \beta} .
$$

It is worth noting that $b_{i j}^{\alpha \beta} \in L^{2}(Y)$ is 1-periodic with $\int_{Y} b_{i j}^{\alpha \beta}(y) d y=0$.

Lemma 2.4. There exist $\Phi_{k i j}^{\alpha \beta} \in H_{p e r}^{1}(Y)$ and $q_{i j}^{\beta} \in H_{p e r}^{1}(Y)$ such that

$$
b_{i j}^{\alpha \beta}=\frac{\partial}{\partial y_{k}}\left(\Phi_{k i j}^{\alpha \beta}\right)+\frac{\partial}{\partial y_{\alpha}}\left(q_{i j}^{\beta}\right) \quad \text { and } \Phi_{k i j}^{\alpha \beta}=-\Phi_{i k j}^{\alpha \beta} .
$$

Moreover,

$$
\left\|\Phi_{k i j}^{\alpha \beta}\right\|_{L^{2}(Y)}+\left\|q_{i j}^{\beta}\right\|_{L^{2}(Y)} \leq C
$$

and with relation

$$
\pi_{j}^{\beta}=\frac{\partial q_{i j}^{\beta}}{\partial y_{i}}
$$

where $C$ depends only on $d$ and $\mu$.

\subsection{Steklov smoothing operator and boundary layer integrals}

Steklov smoothing operator will again play a vital part in this paper, we refer the readers to literature such as [19, 21, 24, 25, 27] and their references for its detailed properties and applications.

We define the Steklov smoothing operator $S_{\varepsilon}$ in $L^{2}\left(\mathbb{R}^{d} ; \mathbb{R}^{d}\right)$ by

$$
\left(S_{\varepsilon} u\right)(x)=f_{Y} u(x-\varepsilon z) d z
$$

which satisfies the estimate $\left\|S_{\varepsilon} u\right\|_{L^{2}\left(\mathbb{R}^{d}\right)} \leq\|u\|_{L^{2}\left(\mathbb{R}^{d}\right)}$. Obviously, $D^{\alpha} S_{\varepsilon} u=S_{\varepsilon} D^{\alpha} u$ for $u \in H^{s}\left(\mathbb{R}^{d} ; \mathbb{R}^{d}\right)$ and any multi-index $\alpha$ such that $|\alpha| \leq s$, and easily we can see that $\left\|S_{\varepsilon} u\right\|_{H^{s}\left(\mathbb{R}^{d}\right)} \leq\|u\|_{H^{s}\left(\mathbb{R}^{d}\right)}$. 
Proposition 2.5. For any $u \in H^{1}\left(\mathbb{R}^{d}\right)$ we have

$$
\left\|S_{\varepsilon} u-u\right\|_{L^{2}\left(\mathbb{R}^{d}\right)} \leq C \varepsilon\|\nabla u\|_{L^{2}\left(\mathbb{R}^{d}\right)},
$$

where $C$ does not depend on $\varepsilon$.

Proposition 2.6. Let $f(x)$ be a 1-periodic function in $\mathbb{R}^{d}$ such that $f \in L^{2}(Y)$. Then for any $u \in L^{2}\left(\mathbb{R}^{d}\right)$,

$$
\left\|f^{\varepsilon} S_{\varepsilon} u\right\|_{L^{2}\left(\mathbb{R}^{d}\right)} \leq\|f\|_{L^{2}(Y)}\|u\|_{L^{2}\left(\mathbb{R}^{d}\right)} .
$$

We define the $r$-neighborhood of the boundary $\partial \Omega$ by

$$
\begin{aligned}
(\partial \Omega)_{r} & =\left\{x \in \mathbb{R}^{d}: \operatorname{dist}(x, \partial \Omega) \leq r\right\}, \\
\Omega_{r} & =\{x \in \Omega: \operatorname{dist}(x, \partial \Omega) \leq r\} .
\end{aligned}
$$

The following lemma gives us an estimate for integrals near the boundary, see [24,25] for example. We will use it repeatedly in the following content.

Lemma 2.7. Let $\Omega \subset \mathbb{R}^{d}$ be a bounded $C^{1}$ domain. Then, for any function $u \in H^{1}(\Omega)$ and for any $0<r \leq \operatorname{diam}(\Omega)$,

$$
\left(\int_{\Omega_{r}}|u|^{2} d x\right)^{1 / 2} \leq C \sqrt{r}\|u\|_{H^{1}(\Omega)}^{1 / 2}\|u\|_{L^{2}(\Omega)}^{1 / 2} .
$$

Moreover, for any 1-periodic function $f \in L^{2}(Y)$ and $u \in H^{1}\left(\mathbb{R}^{d}\right)$,

$$
\left(\int_{(\partial \Omega)_{2 \varepsilon}}\left|f^{\varepsilon}\right|^{2}\left|S_{\varepsilon} u\right|^{2} d x\right)^{1 / 2} \leq C \sqrt{\varepsilon}\|f\|_{L^{2}(Y)}^{1 / 2}\|u\|_{H^{1}\left(\mathbb{R}^{d}\right)}^{1 / 2}\|u\|_{L^{2}\left(\mathbb{R}^{d}\right)}^{1 / 2},
$$

where $C$ depends only on $\Omega$.

\section{Convergence rates for $u_{\varepsilon}$ in $H^{1}$}

From now on we will assume that $\Omega$ is a bounded $C^{1,1}$ domain, and $F \in L^{2}\left(\Omega ; \mathbb{R}^{d}\right)$, $g \in H^{1}(\Omega)$, and $f \in H^{1 / 2}\left(\partial \Omega ; \mathbb{R}^{d}\right)$ satisfy the compatibility condition (1.7). We further assume that $\int_{\Omega} u_{\varepsilon}=\int_{\Omega} u_{0}=0$, and also $\int_{\Omega} p_{\varepsilon}=\int_{\Omega} p_{0}=0$. We define a linear continuous extension operator $E_{\Omega}: H^{2}\left(\Omega ; \mathbb{R}^{d}\right) \rightarrow H^{2}\left(\mathbb{R}^{d} ; \mathbb{R}^{d}\right)$ and for simplicity we denote

$$
\widetilde{u}_{0}=E_{\Omega} u_{0}
$$

so that $\widetilde{u}_{0}=u_{0}$ in $\Omega$ and

$$
\left\|\widetilde{u}_{0}\right\|_{H^{2}\left(\mathbb{R}^{d}\right)} \leq C\left\|u_{0}\right\|_{H^{2}(\Omega)}
$$

where $C$ depends on $\Omega$.

We define $u_{0}+\varepsilon \chi^{\varepsilon} S_{\varepsilon}\left(\nabla \widetilde{u}_{0}\right)$ as a first order approximation of $u_{\varepsilon}$, and let

$$
v_{\varepsilon}=u_{\varepsilon}-u_{0}-\varepsilon \chi^{\varepsilon} S_{\varepsilon}\left(\nabla \widetilde{u}_{0}\right) .
$$

To prove (1.9), we need to show $\left\|v_{\varepsilon}\right\|_{H^{1}(\Omega)} \leq C \sqrt{\varepsilon}\left\|u_{0}\right\|_{H^{2}(\Omega)}$. 
Lemma 3.1. Let $\Omega$ be a bounded $C^{1,1}$ domain. Suppose $A$ satisfies ellipticity condition (1.4) and periodicity condition (1.5). Given $F \in L^{2}\left(\Omega ; \mathbb{R}^{d}\right)$ and $f \in H^{1 / 2}\left(\partial \Omega ; \mathbb{R}^{d}\right)$ satisfying the compatibility condition (1.7), for $g \in H^{1}(\Omega),\left(u_{\varepsilon}, p_{\varepsilon}\right),\left(u_{0}, p_{0}\right)$ are weak solutions of Neumann problems (1.1) and (1.6), respectively. If $\int_{\Omega} u_{\varepsilon}=\int_{\Omega} u_{0}=0$ and $\int_{\Omega} p_{\varepsilon}=\int_{\Omega} p_{0}=0$, and $v_{\varepsilon}$ is defined as in (3.3), then $v_{\varepsilon}$ satisfies the following weak formulation

$$
\begin{aligned}
a_{\varepsilon}\left(v_{\varepsilon}, \varphi\right)= & \int_{\Omega}\left[p_{\varepsilon}-p_{0}\right] \operatorname{div}(\varphi)-\int_{\Omega}\left[\widehat{A}-A^{\varepsilon}\right]\left[S_{\varepsilon}\left(\nabla \widetilde{u}_{0}\right)-\nabla u_{0}\right] \cdot \nabla \varphi \\
& -\varepsilon \int_{\Omega} A^{\varepsilon} \chi^{\varepsilon} S_{\varepsilon}\left(\nabla^{2} \widetilde{u}_{0}\right) \cdot \nabla \varphi-\int_{\Omega} B^{\varepsilon} S_{\varepsilon}\left(\nabla \widetilde{u}_{0}\right) \cdot \nabla \varphi, \quad \forall \varphi \in H^{1}\left(\Omega ; \mathbb{R}^{d}\right) .
\end{aligned}
$$

Proof. Since $\left(u_{\varepsilon}, p_{\varepsilon}\right),\left(u_{0}, p_{0}\right)$ satisfy the weak formulations (2.2) and (2.7), respectively, therefore for any $\varphi \in H^{1}\left(\Omega ; \mathbb{R}^{d}\right)$,

$$
a_{\varepsilon}\left(u_{\varepsilon}, \varphi\right)-\int_{\Omega} p_{\varepsilon} \operatorname{div}(\varphi)=\int_{\Omega} \widehat{a}_{i j}^{\alpha \beta} \frac{\partial u_{0}^{\beta}}{\partial x_{j}} \frac{\partial \varphi^{\alpha}}{\partial x_{i}}-\int_{\Omega} p_{0} \operatorname{div}(\varphi) .
$$

By (3.5) and the definition of $v_{\varepsilon}$, we have

$$
\begin{aligned}
a_{\varepsilon}\left(v_{\varepsilon}, \varphi\right) & =a_{\varepsilon}\left(u_{\varepsilon}, \varphi\right)-a_{\varepsilon}\left(u_{0}, \varphi\right)-a_{\varepsilon}\left(\varepsilon \chi^{\varepsilon} S_{\varepsilon}\left(\nabla \widetilde{u}_{0}\right), \varphi\right) \\
& =\int_{\Omega}\left(p_{\varepsilon}-p_{0}\right) \operatorname{div}(\varphi)+\int_{\Omega}\left[\widetilde{a}_{i j}^{\alpha \beta}-a_{i j}^{\alpha \beta}(x / \varepsilon)\right] \frac{\partial u_{0}^{\beta}}{\partial x_{j}} \frac{\partial \varphi^{\alpha}}{\partial x_{i}}-a_{\varepsilon}\left(\varepsilon \chi^{\varepsilon} S_{\varepsilon}\left(\nabla \widetilde{u}_{0}\right), \varphi\right) .
\end{aligned}
$$

Since

$$
\begin{aligned}
a_{\varepsilon}\left(\varepsilon \chi^{\varepsilon} S_{\varepsilon}\left(\nabla \widetilde{u}_{0}\right), \varphi\right)=\int_{\Omega} a_{i k}^{\alpha \gamma}(x / \varepsilon) \frac{\partial}{\partial x_{k}} & \left(\varepsilon \chi_{j}^{\gamma \beta}(x / \varepsilon)\right) S_{\varepsilon} \frac{\partial \widetilde{u}_{0}^{\beta}}{\partial x_{j}} \frac{\partial \varphi^{\alpha}}{\partial x_{i}} \\
& +\varepsilon \int_{\Omega} a_{i k}^{\alpha \gamma}(x / \varepsilon) \chi_{j}^{\gamma \beta}(x / \varepsilon) S_{\varepsilon} \frac{\partial^{2} \widetilde{u}_{0}^{\beta}}{\partial x_{k} \partial x_{j}} \frac{\partial \varphi^{\alpha}}{\partial x_{i}}
\end{aligned}
$$

then by using the definition of $b$, we can obtain that

$$
\begin{aligned}
a_{\varepsilon}\left(v_{\varepsilon}, \varphi\right)= & \int_{\Omega}\left(p_{\varepsilon}-p_{0}\right) \operatorname{div}(\varphi)-\int_{\Omega}\left[\widehat{a}_{i j}^{\alpha \beta}-a_{i j}^{\alpha \beta}(x / \varepsilon)\right]\left[S_{\varepsilon} \frac{\partial \widetilde{u}_{0}^{\beta}}{\partial x_{j}}-\frac{\partial u_{0}^{\beta}}{\partial x_{j}}\right] \frac{\partial \varphi^{\alpha}}{\partial x_{i}} \\
& -\varepsilon \int_{\Omega} a_{i k}^{\alpha \gamma}(x / \varepsilon) \chi_{j}^{\gamma \beta}(x / \varepsilon) S_{\varepsilon} \frac{\partial^{2} \widetilde{u}_{0}^{\beta}}{\partial x_{k} \partial x_{j}} \frac{\partial \varphi^{\alpha}}{\partial x_{i}}-\int_{\Omega} b_{i j}^{\alpha \beta}(x / \varepsilon) S_{\varepsilon} \frac{\partial \widetilde{u}_{0}^{\beta}}{\partial x_{j}} \frac{\partial \varphi^{\alpha}}{\partial x_{i}}
\end{aligned}
$$

We choose a cut-off function $\theta_{\varepsilon}(x)$ in $\mathbb{R}^{d}$ satisfying the following conditions,

$$
\begin{aligned}
& \theta_{\varepsilon} \in C_{0}^{\infty}\left(\mathbb{R}^{d}\right), \quad \operatorname{supp}\left(\theta_{\varepsilon}\right) \subset(\partial \Omega)_{\varepsilon}, \quad 0 \leq \theta_{\varepsilon}(x) \leq 1, \\
& \left.\theta_{\varepsilon}\right|_{\partial \Omega}=1, \quad\left|\nabla \theta_{\varepsilon}\right| \leq \kappa / \varepsilon .
\end{aligned}
$$

The following lemma is the key intermediate step to prove our convergence results. 
Lemma 3.2. Let $v_{\varepsilon}$ be defined as in (3.3). Then, for any $\varphi \in H^{1}\left(\Omega ; \mathbb{R}^{d}\right)$ we have

$$
\left|a_{\varepsilon}\left(v_{\varepsilon}, \varphi\right)\right| \leq C\left\|u_{0}\right\|_{H^{2}(\Omega)}\left[\|\operatorname{div}(\varphi)\|_{L^{2}(\Omega)}+\varepsilon^{1 / 2}\|\nabla \varphi\|_{L^{2}\left(\Omega_{2 \varepsilon}\right)}+\varepsilon\|\nabla \varphi\|_{L^{2}(\Omega)}\right],
$$

where the constant $C$ depends only on $\mu, d$, and $\Omega$.

Proof. For any $\varphi \in H^{1}\left(\Omega ; \mathbb{R}^{d}\right)$, by Lemma 3.1, we have proved that $v_{\varepsilon}$ satisfies the weak formulation (3.8). By Lemma 2.4, we may rewrite the last term in RHS of (3.8) as

$$
-\int_{\Omega} \frac{\partial}{\partial x_{k}}\left(\varepsilon \Phi_{k i j}^{\alpha \beta}(x / \varepsilon)\right) S_{\varepsilon} \frac{\partial \widetilde{u}_{0}^{\beta}}{\partial x_{j}} \frac{\partial \varphi^{\alpha}}{\partial x_{i}}-\int_{\Omega} \frac{\partial}{\partial x_{\alpha}}\left(\varepsilon q_{i j}^{\beta}(x / \varepsilon)\right) S_{\varepsilon} \frac{\partial \widetilde{u}_{0}^{\beta}}{\partial x_{j}} \frac{\partial \varphi^{\alpha}}{\partial x_{i}}=R_{1}+R_{2} .
$$

For the first integral $R_{1}$, we see that

$$
R_{1}=-\varepsilon \int_{\Omega} \frac{\partial}{\partial x_{k}}\left(\Phi_{k i j}^{\alpha \beta}(x / \varepsilon) S_{\varepsilon} \frac{\partial \widetilde{u}_{0}^{\beta}}{\partial x_{j}}\right) \frac{\partial \varphi^{\alpha}}{\partial x_{i}}+\varepsilon \int_{\Omega} \Phi_{k i j}^{\alpha \beta}(x / \varepsilon) S_{\varepsilon} \frac{\partial^{2} \widetilde{u}_{0}^{\beta}}{\partial x_{k} \partial x_{j}} \frac{\partial \varphi^{\alpha}}{\partial x_{i}}=R_{1 a}+R_{1 b} .
$$

For the term $R_{1 a}$, recall that $\Phi$ is anti-symmetric and $\theta_{\varepsilon} \equiv 1$ on $\partial \Omega$, therefore

$$
\begin{aligned}
& R_{1 a}=-\varepsilon \int_{\Omega} \frac{\partial}{\partial x_{k}}\left(\left[\theta_{\varepsilon}+1-\theta_{\varepsilon}\right] \Phi_{k i j}^{\alpha \beta}(x / \varepsilon) S_{\varepsilon} \frac{\partial \widetilde{u}_{0}^{\beta}}{\partial x_{j}}\right) \frac{\partial \varphi^{\alpha}}{\partial x_{i}} \\
& =-\varepsilon \int_{\Omega} \frac{\partial}{\partial x_{k}}\left(\theta_{\varepsilon} \Phi_{k i j}^{\alpha \beta}(x / \varepsilon) S_{\varepsilon} \frac{\partial \widetilde{u}_{0}^{\beta}}{\partial x_{j}}\right) \frac{\partial \varphi^{\alpha}}{\partial x_{i}}+\varepsilon \int_{\Omega} \frac{\partial^{2}}{\partial x_{k} \partial x_{i}}\left(\left(1-\theta_{\varepsilon}\right) \Phi_{k i j}^{\alpha \beta}(x / \varepsilon) S_{\varepsilon} \frac{\partial \widetilde{u}_{0}^{\beta}}{\partial x_{j}}\right) \varphi^{\alpha} \\
& =-\varepsilon \int_{\Omega} \frac{\partial}{\partial x_{k}}\left(\theta_{\varepsilon} \Phi_{k i j}^{\alpha \beta}(x / \varepsilon) S_{\varepsilon} \frac{\partial \widetilde{u}_{0}^{\beta}}{\partial x_{j}}\right) \frac{\partial \varphi^{\alpha}}{\partial x_{i}} .
\end{aligned}
$$

Similarly for $R_{2}$, we write it as

$$
R_{2}=-\varepsilon \int_{\Omega} \frac{\partial}{\partial x_{\alpha}}\left(q_{i j}^{\beta}(x / \varepsilon) S_{\varepsilon} \frac{\partial \widetilde{u}_{0}^{\beta}}{\partial x_{j}}\right) \frac{\partial \varphi^{\alpha}}{\partial x_{i}}+\varepsilon \int_{\Omega} q_{i j}^{\beta}(x / \varepsilon) S_{\varepsilon} \frac{\partial^{2} \widetilde{u}_{0}^{\beta}}{\partial x_{k} \partial x_{j}} \frac{\partial \varphi^{\alpha}}{\partial x_{i}}=R_{2 a}+R_{2 b}
$$

and analogously for the term $R_{2 a}$, since $\theta_{\varepsilon} \equiv 1$ on $\partial \Omega$,

$$
\begin{aligned}
& R_{2 a}=-\varepsilon \int_{\Omega} \frac{\partial}{\partial x_{\alpha}}\left(\left[\theta_{\varepsilon}+1-\theta_{\varepsilon}\right] q_{i j}^{\beta}(x / \varepsilon) S_{\varepsilon} \frac{\partial \widetilde{u}_{0}^{\beta}}{\partial x_{j}}\right) \frac{\partial \varphi^{\alpha}}{\partial x_{i}} \\
& =-\varepsilon \int_{\Omega} \frac{\partial}{\partial x_{\alpha}}\left(\theta_{\varepsilon} q_{i j}^{\beta}(x / \varepsilon) S_{\varepsilon} \frac{\partial \widetilde{u}_{0}^{\beta}}{\partial x_{j}}\right) \frac{\partial \varphi^{\alpha}}{\partial x_{i}}+\varepsilon \int_{\Omega} \frac{\partial^{2}}{\partial x_{\alpha} \partial x_{i}}\left(\left(1-\theta_{\varepsilon}\right) q_{i j}^{\beta}(x / \varepsilon) S_{\varepsilon} \frac{\partial \widetilde{u}_{0}^{\beta}}{\partial x_{j}}\right) \varphi^{\alpha} \\
& =-\varepsilon \int_{\Omega} \frac{\partial}{\partial x_{\alpha}}\left(\theta_{\varepsilon} q_{i j}^{\beta}(x / \varepsilon) S_{\varepsilon} \frac{\partial \widetilde{u}_{0}^{\beta}}{\partial x_{j}}\right) \frac{\partial \varphi^{\alpha}}{\partial x_{i}}-\varepsilon \int_{\Omega} \frac{\partial}{\partial x_{i}}\left(\left(1-\theta_{\varepsilon}\right) q_{i j}^{\beta}(x / \varepsilon) S_{\varepsilon} \frac{\partial \widetilde{u}_{0}^{\beta}}{\partial x_{j}}\right) \operatorname{div}(\varphi) .
\end{aligned}
$$


By (2.11), it is more precise that

$$
\begin{aligned}
R_{2 a} & =\int_{\Omega}\left[\left(\varepsilon \frac{\partial \theta_{\varepsilon}}{\partial x_{i}} q_{i j}^{\beta}(x / \varepsilon)-\left(1-\theta_{\varepsilon}\right) \pi_{j}^{\beta}(x / \varepsilon)\right) S_{\varepsilon} \frac{\partial \widetilde{u}_{0}^{\beta}}{\partial x_{j}}-\varepsilon\left(1-\theta_{\varepsilon}\right) q_{i j}^{\beta}(x / \varepsilon) S_{\varepsilon} \frac{\partial^{2} \widetilde{u}_{0}^{\beta}}{\partial x_{i} \partial x_{j}}\right] \operatorname{div}(\varphi) \\
& -\varepsilon \int_{\Omega} \frac{\partial}{\partial x_{\alpha}}\left(\theta_{\varepsilon} q_{i j}^{\beta}(x / \varepsilon) S_{\varepsilon} \frac{\partial \widetilde{u}_{0}^{\beta}}{\partial x_{j}}\right) \frac{\partial \varphi^{\alpha}}{\partial x_{i}} .
\end{aligned}
$$

Therefore, we have updated (3.8) as

$$
a_{\varepsilon}\left(v_{\varepsilon}, \varphi\right)=I_{1}[\varphi]+I_{2}[\varphi]+I_{3}[\varphi]
$$

where $I_{1}, I_{2}$, and $I_{3}$ are defined as the following,

$$
\begin{aligned}
I_{1}[\varphi]= & \int_{\Omega}\left[p_{\varepsilon}-p_{0}-\left[\left(1-\theta_{\varepsilon}\right) \pi^{\varepsilon}-\varepsilon \nabla \theta_{\varepsilon} q^{\varepsilon}\right] S_{\varepsilon}\left(\nabla \widetilde{u}_{0}\right)-\varepsilon\left(1-\theta_{\varepsilon}\right) q^{\varepsilon} S_{\varepsilon}\left(\nabla^{2} \widetilde{u}_{0}\right)\right] \operatorname{div}(\varphi) ; \\
I_{2}[\varphi]= & \varepsilon \int_{\Omega}\left[q^{\varepsilon} S_{\varepsilon}\left(\nabla^{2} \widetilde{u}_{0}\right)\right] \cdot \nabla \varphi+\varepsilon \int_{\Omega}\left[\left(\Phi^{\varepsilon}-A^{\varepsilon} \chi^{\varepsilon}\right) S_{\varepsilon}\left(\nabla^{2} \widetilde{u}_{0}\right)\right] \cdot \nabla \varphi \\
& -\int_{\Omega}\left[\left(\widehat{A}-A^{\varepsilon}\right)\left(S_{\varepsilon}\left(\nabla \widetilde{u}_{0}\right)-\nabla u_{0}\right)\right] \cdot \nabla \varphi ; \\
I_{3}[\varphi]= & -\int_{\Omega}\left[\nabla\left(\varepsilon \theta_{\varepsilon} \Phi^{\varepsilon} S_{\varepsilon}\left(\nabla \widetilde{u}_{0}\right)\right)\right] \cdot \nabla \varphi-\int_{\Omega}\left[\nabla\left(\varepsilon \theta_{\varepsilon} q^{\varepsilon} S_{\varepsilon}\left(\nabla \widetilde{u}_{0}\right)\right)\right] \cdot \nabla \varphi .
\end{aligned}
$$

To estimate $I_{1}$, since $\left(v_{\varepsilon}, p_{\varepsilon}-p_{0}\right)$ satisfies the Stokes system (3.4) with data only involves with the gradient of $u_{0}$, therefore by energy estimate (2.3), Propositions 2.5 2.6 and the assumption that $\int_{\Omega} p_{\varepsilon}=\int_{\Omega} p_{0}=0$, we obtain that

$$
\left\|p_{\varepsilon}-p_{0}\right\|_{L^{2}(\Omega)} \leq C\left\|u_{0}\right\|_{H^{2}(\Omega)} .
$$

Since $\pi \in L^{2}(Y), q \in H^{1}\left(Y ; \mathbb{R}^{d}\right)$ and $\left|\nabla \theta_{\varepsilon}\right| \leq \kappa / \varepsilon$, by Proposition 2.6, Hölder's inequality and (3.19), we have

$$
\left|I_{1}[\varphi]\right| \leq C\left\|u_{0}\right\|_{H^{2}(\Omega)}\|\operatorname{div}(\varphi)\|_{L^{2}(\Omega)},
$$

where $C$ depends only on $d, \mu$, and $\Omega$.

By Propositions 2.5] 2.6, (3.2), and Hölder's inequality, we can obtain that

$$
\begin{aligned}
\left|I_{2}[\varphi]\right| & \leq C \varepsilon\left(\left[\|\chi\|_{L^{2}(Y)}+\|\Phi\|_{L^{2}(Y)}+\|q\|_{L^{2}(Y)}+1\right]\left\|\widetilde{u}_{0}\right\|_{H^{2}\left(\mathbb{R}^{d}\right)}\right)\|\nabla \varphi\|_{L^{2}(\Omega)} \\
& \leq C \varepsilon\left\|u_{0}\right\|_{H^{2}(\Omega)}\|\nabla \varphi\|_{L^{2}(\Omega)} .
\end{aligned}
$$

For $I_{3}$, since $\operatorname{supp} \theta_{\varepsilon} \subset(\partial \Omega)_{\varepsilon}$ and again by Hölder's inequality, we have

$$
\left|I_{3}[\varphi]\right| \leq C\left(\left\|\varepsilon \theta_{\varepsilon} q^{\varepsilon} S_{\varepsilon}\left(\nabla \widetilde{u}_{0}\right)\right\|_{H^{1}(\Omega)}+\left\|\varepsilon \theta_{\varepsilon} \Phi^{\varepsilon} S_{\varepsilon}\left(\nabla \widetilde{u}_{0}\right)\right\|_{H^{1}(\Omega)}\right)\|\nabla \varphi\|_{L^{2}\left(\Omega_{2 \varepsilon}\right)} .
$$


For the first term in the parentheses of (3.22),

$$
\begin{aligned}
\varepsilon\left\|\theta_{\varepsilon} q^{\varepsilon} S_{\varepsilon}\left(\nabla \widetilde{u}_{0}\right)\right\|_{H^{1}(\Omega)} \leq & C \varepsilon\left\{\left\|q^{\varepsilon} S_{\varepsilon}\left(\nabla \widetilde{u}_{0}\right)\right\|_{L^{2}(\Omega)}+\left\|\left(\nabla \theta_{\varepsilon}\right) q^{\varepsilon} S_{\varepsilon}\left(\nabla \widetilde{u}_{0}\right)\right\|_{L^{2}(\Omega)}\right. \\
& \left.+\varepsilon^{-1}\left\|\theta_{\varepsilon}(\nabla q)^{\varepsilon} S_{\varepsilon}\left(\nabla \widetilde{u}_{0}\right)\right\|_{L^{2}(\Omega)}+\left\|q^{\varepsilon} S_{\varepsilon}\left(\nabla^{2} \widetilde{u}_{0}\right)\right\|_{L^{2}(\Omega)}\right\} \\
\leq & C \varepsilon\left\{\left\|\widetilde{u}_{0}\right\|_{H^{2}\left(\mathbb{R}^{d}\right)}+\varepsilon^{-1}\left\|q^{\varepsilon} S_{\varepsilon}\left(\nabla \widetilde{u}_{0}\right)\right\|_{L^{2}\left((\partial \Omega)_{\varepsilon}\right)}\right. \\
& \left.+\varepsilon^{-1}\left\|(\nabla q)^{\varepsilon} S_{\varepsilon}\left(\nabla \widetilde{u}_{0}\right)\right\|_{L^{2}\left((\partial \Omega)_{\varepsilon}\right)}\right\} \\
\leq & C \varepsilon^{1 / 2}\left\|\widetilde{u}_{0}\right\|_{H^{2}\left(\mathbb{R}^{d}\right)},
\end{aligned}
$$

where we have used Proposition 2.6 for the second inequality and Lemma 2.7 for the last. Using the same manner, we can show that

$$
\varepsilon\left\|\theta_{\varepsilon} \Phi^{\varepsilon} S_{\varepsilon}\left(\nabla \widetilde{u}_{0}\right)\right\|_{H^{1}(\Omega)} \leq C \varepsilon^{1 / 2}\left\|\widetilde{u}_{0}\right\|_{H^{2}\left(\mathbb{R}^{d}\right)} .
$$

Therefore, by (3.2), we have proved that

$$
\left|I_{3}[\varphi]\right| \leq C \varepsilon^{1 / 2}\left\|u_{0}\right\|_{H^{2}(\Omega)}\|\nabla \varphi\|_{L^{2}\left(\Omega_{2 \varepsilon}\right)}
$$

Hence, by combining (3.20), (3.21) and (3.25), we know that

$$
\left|a_{\varepsilon}\left(v_{\varepsilon}, \varphi\right)\right| \leq C\left\|u_{0}\right\|_{H^{2}(\Omega)}\left[\|\operatorname{div}(\varphi)\|_{L^{2}(\Omega)}+\varepsilon^{1 / 2}\|\nabla \varphi\|_{L^{2}\left(\Omega_{2 \varepsilon}\right)}+\varepsilon\|\nabla \varphi\|_{L^{2}(\Omega)}\right],
$$

where $C$ depends only on $d, \mu$, and $\Omega$.

Proof of estimate (1.9). We will now prove (1.9) by energy estimates. Since $\operatorname{div}\left(u_{\varepsilon}\right)=$ $\operatorname{div}\left(u_{0}\right)$, and recall that $\operatorname{div}(\chi)=0$, hence

$$
\operatorname{div}\left(v_{\varepsilon}\right)=-\varepsilon \chi_{j}^{\alpha \beta}(x / \varepsilon) S_{\varepsilon} \frac{\partial^{2} \widetilde{u}_{0}^{\beta}}{\partial x_{\alpha} \partial x_{j}} .
$$

By Proposition 2.6, we can see that

$$
\left\|\operatorname{div}\left(v_{\varepsilon}\right)\right\|_{L^{2}(\Omega)} \leq C \varepsilon\left\|u_{0}\right\|_{H^{2}(\Omega)}
$$

If we choose $\varphi=v_{\varepsilon}$ itself in Lemma 3.2, therefore by ellipticity condition (1.4), (3.10) and (3.26), we see that

$$
\left\|\nabla v_{\varepsilon}\right\|_{L^{2}(\Omega)}^{2} \leq C\left\|u_{0}\right\|_{H^{2}(\Omega)}\left[\varepsilon^{1 / 2}\left\|\nabla v_{\varepsilon}\right\|_{L^{2}\left(\Omega_{2 \varepsilon}\right)}+\varepsilon\left\|\nabla v_{\varepsilon}\right\|_{L^{2}(\Omega)}\right]+C \varepsilon\left\|u_{0}\right\|_{H^{2}(\Omega)}^{2} .
$$

By Poincaré's inequality, and since $\int_{\Omega} u_{\varepsilon}=\int_{\Omega} u_{0}=0$, we have

$$
\begin{aligned}
\left\|v_{\varepsilon}\right\|_{L^{2}(\Omega)} & \leq\left\|v_{\varepsilon}-f_{\Omega} v_{\varepsilon}\right\|_{L^{2}(\Omega)}+\left\|f_{\Omega}\left[\varepsilon \chi^{\varepsilon} S_{\varepsilon}\left(\nabla \widetilde{u}_{0}\right)\right]\right\|_{L^{2}(\Omega)} \\
& \leq C\left\|\nabla v_{\varepsilon}\right\|_{L^{2}(\Omega)}+C \varepsilon\left\|u_{0}\right\|_{H^{2}(\Omega)},
\end{aligned}
$$

where we have also used Proposition 2.6 and (3.2) for the last inequality. Finally, by (3.27), (3.28) and Cauchy-Schwarz inequality, we proved the desired result

$$
\left\|v_{\varepsilon}\right\|_{H^{1}(\Omega)} \leq C \sqrt{\varepsilon}\left\|u_{0}\right\|_{H^{2}(\Omega)},
$$

where $C$ depends only on $d, \mu$, and $\Omega$. 
Remark 3.3. This key intermediate step (3.10) has a nature advantage in the Neumann boundary value problems, because the boundary integral of the weak formulation no longer contains the pressure term. But as we mentioned earlier, Neumann problems are always more complicated than Dirichlet problems. Hence, with a slight modification, this method is also suitable for the Dirichlet convergence rate problem as we did in [8]. We need to choose a Dirichlet boundary corrector $\left(w_{\varepsilon}, \tau_{\varepsilon}\right)$, which satisfies $\mathcal{L}_{\varepsilon}\left(w_{\varepsilon}\right)+\nabla \tau_{\varepsilon}=0, \operatorname{div}\left(w_{\varepsilon}\right)=$ $\varepsilon \operatorname{div}\left(\chi^{\varepsilon} S_{\varepsilon} \nabla \widetilde{u}_{0}\right)$ and $\left.w_{\varepsilon}\right|_{\partial \Omega}=\varepsilon \chi^{\varepsilon} S_{\varepsilon} \nabla \widetilde{u}_{0}$. Let $z_{\varepsilon}=u_{\varepsilon}-u_{0}-\varepsilon \chi^{\varepsilon} S_{\varepsilon} \nabla \widetilde{u}_{0}+w_{\varepsilon}$, since $z_{\varepsilon}$ belongs to the Hilbert space $V=\left\{u \in H_{0}^{1}\left(\Omega ; \mathbb{R}^{d}\right): \operatorname{div}(u)=0\right\}$, it is not hard to see that $\left|a_{\varepsilon}\left(z_{\varepsilon}, \varphi\right)\right| \leq C \varepsilon\left\|u_{0}\right\|_{H^{2}(\Omega)}\|\nabla \varphi\|_{L^{2}(\Omega)}$, for any $\varphi \in V$. Therefore the $O(\sqrt{\varepsilon})$ rate can be obtained by choosing $\varphi=z_{\varepsilon}$ and the fact that $\left\|w_{\varepsilon}\right\|_{H^{1}(\Omega)} \leq C \sqrt{\varepsilon}\left\|u_{0}\right\|_{H^{2}(\Omega)}$. This gives us another aspect of seeing the importance of the choice of boundary corrector $w_{\varepsilon}$ in [8].

\section{Convergence rates of $p_{\varepsilon}$ in $L^{2}$}

It is well known that if $\left(u_{\varepsilon}, p_{\varepsilon}\right) \in H^{1}\left(\Omega ; \mathbb{R}^{d}\right) \times L^{2}(\Omega)$ is a weak solution of any Stokes system (2.1), then

$$
\left\|p_{\varepsilon}-f_{\Omega} p_{\varepsilon}\right\|_{L^{2}(\Omega)} \leq\left\|\nabla p_{\varepsilon}\right\|_{H^{-1}(\Omega)} \leq C\left\{\|F\|_{H^{-1}(\Omega)}+\left\|u_{\varepsilon}\right\|_{H^{1}(\Omega)}\right\}
$$

where $C$ depends only on $d, \mu$, and $\Omega$ (see e.g. [26]).

Proof of estimate (1.10). Recalling the definition of $b$, we see that

$$
\begin{aligned}
& \left(\mathcal{L}_{\varepsilon}\left(v_{\varepsilon}\right)\right)^{\alpha}=-\frac{\partial\left[p_{\varepsilon}-p_{0}\right]}{\partial x_{\alpha}}-\frac{\partial}{\partial x_{i}}\left(\left[\widehat{a}_{i j}^{\alpha \beta}-a_{i j}^{\alpha \beta}(x / \varepsilon)\right] \frac{\partial u_{0}^{\beta}}{\partial x_{j}}\right) \\
& \quad+\frac{\partial}{\partial x_{i}}\left(a_{i k}^{\alpha \gamma}(x / \varepsilon) \frac{\partial}{\partial x_{k}}\left[\varepsilon \chi_{j}^{\gamma \beta}(x / \varepsilon)\right] S_{\varepsilon} \frac{\partial \widetilde{u}_{0}^{\beta}}{\partial x_{j}}\right)+\varepsilon \frac{\partial}{\partial x_{i}}\left(a_{i k}^{\alpha \gamma}(x / \varepsilon) \chi_{j}^{\gamma \beta}(x / \varepsilon) S_{\varepsilon} \frac{\partial^{2} \tilde{u}_{0}^{\beta}}{\partial x_{k} \partial x_{j}}\right) \\
& =-\frac{\partial\left[p_{\varepsilon}-p_{0}\right]}{\partial x_{\alpha}}+\frac{\partial}{\partial x_{i}}\left(\left[\widehat{a}_{i j}^{\alpha \beta}-a_{i j}^{\alpha \beta}(x / \varepsilon)\right]\left[S_{\varepsilon} \frac{\partial \widetilde{u}_{0}^{\beta}}{\partial x_{j}}-\frac{\partial u_{0}^{\beta}}{\partial x_{j}}\right]\right) \\
& \quad+\varepsilon \frac{\partial}{\partial x_{i}}\left(a_{i k}^{\alpha \gamma}(x / \varepsilon) \chi_{j}^{\gamma \beta}(x / \varepsilon) S_{\varepsilon} \frac{\partial^{2} \widetilde{u}_{0}^{\beta}}{\partial x_{k} \partial x_{j}}\right)+\frac{\partial}{\partial x_{i}}\left(b_{i j}^{\alpha \beta}(x / \varepsilon) S_{\varepsilon} \frac{\partial \widetilde{u}_{0}^{\beta}}{\partial x_{j}}\right) .
\end{aligned}
$$

Using Lemma 2.4, we'd split the last term of RHS of (4.2) into two,

$$
\begin{aligned}
\frac{\partial}{\partial x_{i}}\left(b_{i j}^{\alpha \beta}(x / \varepsilon) S_{\varepsilon} \frac{\partial \tilde{u}_{0}^{\beta}}{\partial x_{j}}\right) & =\frac{\partial}{\partial x_{i}}\left(\left[\frac{\partial}{\partial x_{k}}\left(\varepsilon \Phi_{k i j}^{\alpha \beta}(x / \varepsilon)\right)+\frac{\partial}{\partial x_{\alpha}}\left(\varepsilon q_{i j}^{\beta}(x / \varepsilon)\right)\right] S_{\varepsilon} \frac{\partial \widetilde{u}_{0}^{\beta}}{\partial x_{j}}\right) \\
& =K_{1}+K_{2} .
\end{aligned}
$$


Because of the anti-symmetry property $\Phi_{k i j}^{\alpha \beta}=-\Phi_{i k j}^{\alpha \beta}$, we see that

$$
\begin{aligned}
K_{1} & =\frac{\partial^{2}}{\partial x_{i} \partial x_{k}}\left(\varepsilon \Phi_{k i j}^{\alpha \beta}(x / \varepsilon) S_{\varepsilon} \frac{\partial{\widetilde{u_{0}}}^{\beta}}{\partial x_{j}}\right)-\varepsilon \frac{\partial}{\partial x_{i}}\left(\Phi_{k i j}^{\alpha \beta}(x / \varepsilon) S_{\varepsilon} \frac{\partial^{2} \widetilde{u}_{0}^{\beta}}{\partial x_{j} \partial x_{k}}\right) \\
& =-\varepsilon \frac{\partial}{\partial x_{i}}\left(\Phi_{k i j}^{\alpha \beta}(x / \varepsilon) S_{\varepsilon} \frac{\partial^{2} \widetilde{u}_{0}^{\beta}}{\partial x_{j} \partial x_{k}}\right) .
\end{aligned}
$$

For the second term in the RHS of (4.3), we have

$$
\begin{aligned}
K_{2} & =\frac{\partial}{\partial x_{\alpha}}\left(\frac{\partial}{\partial x_{i}}\left[\varepsilon q_{i j}^{\beta}(x / \varepsilon) S_{\varepsilon} \frac{\partial \widetilde{u}_{0}^{\beta}}{\partial x_{j}}\right]\right)-\frac{\partial}{\partial x_{i}}\left(\varepsilon q_{i j}^{\beta}(x / \varepsilon) S_{\varepsilon} \frac{\partial^{2} \widetilde{u}_{0}^{\beta}}{\partial x_{\alpha} \partial x_{j}}\right) \\
& =K_{3}-\frac{\partial}{\partial x_{i}}\left(\varepsilon q_{i j}^{\beta}(x / \varepsilon) S_{\varepsilon} \frac{\partial^{2} \widetilde{u}_{0}^{\beta}}{\partial x_{\alpha} \partial x_{j}}\right) .
\end{aligned}
$$

In view of (2.11), for the first term on the RHS of (4.4), we obtain

$$
K_{3}=\frac{\partial}{\partial x_{\alpha}}\left(\pi_{j}^{\beta}(x / \varepsilon) S_{\varepsilon} \frac{\partial \widetilde{u}_{0}^{\beta}}{\partial x_{j}}\right)+\frac{\partial}{\partial x_{\alpha}}\left(\varepsilon q_{i j}^{\beta}(x / \varepsilon) S_{\varepsilon} \frac{\partial^{2} \widetilde{u}_{0}^{\beta}}{\partial x_{j} \partial x_{i}}\right) .
$$

We have shown that

$$
\begin{aligned}
\left(\mathcal{L}_{\varepsilon}\left(v_{\varepsilon}\right)\right)^{\alpha} & +\frac{\partial}{\partial x_{\alpha}}\left(p_{\varepsilon}-p_{0}-\pi_{j}^{\beta}(x / \varepsilon) S_{\varepsilon} \frac{\partial \widetilde{u}_{0}^{\beta}}{\partial x_{j}}-\varepsilon q_{i j}^{\beta}(x / \varepsilon) S_{\varepsilon} \frac{\partial^{2} \widetilde{u}_{0}^{\beta}}{\partial x_{j} \partial x_{i}}\right) \\
=\varepsilon \frac{\partial}{\partial x_{i}}\left(\left[a_{i j}^{\alpha \gamma}(x / \varepsilon) \chi_{k}^{\gamma \beta}(x / \varepsilon)-\Phi_{k i j}^{\alpha \beta}(x / \varepsilon)\right] S_{\varepsilon} \frac{\partial^{2} \widetilde{u}_{0}^{\beta}}{\partial x_{j} \partial x_{k}}\right) & \\
& -\varepsilon \frac{\partial}{\partial x_{i}}\left(q_{i j}^{\beta}(x / \varepsilon) S_{\varepsilon} \frac{\partial^{2} \widetilde{u}_{0}^{\beta}}{\partial x_{\alpha} \partial x_{j}}\right)-\frac{\partial}{\partial x_{i}}\left(\left[\widehat{a}_{i j}^{\alpha \beta}-a_{i j}^{\alpha \beta}(x / \varepsilon)\right]\left[\frac{\partial u_{0}^{\beta}}{\partial x_{j}}-S_{\varepsilon} \frac{\partial \widetilde{u}_{0}^{\beta}}{\partial x_{j}}\right]\right) .
\end{aligned}
$$

By applying (4.1) to (4.6), and since $\int_{\Omega} p_{\varepsilon}=\int_{\Omega} p_{0}=0$, we see that

$$
\begin{aligned}
& \left\|\left[p_{\varepsilon}-p_{0}-\pi^{\varepsilon} S_{\varepsilon}\left(\nabla \widetilde{u}_{0}\right)-\varepsilon q^{\varepsilon} S_{\varepsilon}\left(\nabla^{2} \widetilde{u}_{0}\right)\right]+f_{\Omega}\left[\pi^{\varepsilon} S_{\varepsilon}\left(\nabla \widetilde{u}_{0}\right)+\varepsilon q^{\varepsilon} S_{\varepsilon}\left(\nabla^{2} \widetilde{u}_{0}\right)\right]\right\|_{L^{2}(\Omega)} \\
& \leq\left\|\nabla\left[p_{\varepsilon}-p_{0}-\pi^{\varepsilon} S_{\varepsilon}\left(\nabla \widetilde{u}_{0}\right)-\varepsilon q^{\varepsilon} S_{\varepsilon}\left(\nabla^{2} \widetilde{u}_{0}\right)\right]\right\|_{H^{-1}(\Omega)} \\
& \leq C\left\|v_{\varepsilon}\right\|_{H^{1}(\Omega)}+C \varepsilon\left[\|\chi\|_{L^{2}(Y)}+\|\Phi\|_{L^{2}(Y)}+\|q\|_{L^{2}(Y)}+1\right]\left\|\widetilde{u}_{0}\right\|_{H^{2}\left(\mathbb{R}^{d}\right)} \\
& \leq C \sqrt{\varepsilon}\left\|u_{0}\right\|_{H^{2}(\Omega)},
\end{aligned}
$$

where we have used Propositions 2.5 2.6 for the next to last inequality, (1.9) and (3.2) for the last, and the constant $C$ is independent of $\varepsilon$. By Proposition 2.6 and (3.2), we see that

$$
\varepsilon\left\|q^{\varepsilon} S_{\varepsilon}\left(\nabla^{2} \widetilde{u}_{0}\right)-f q^{\varepsilon} S_{\varepsilon}\left(\nabla^{2} \widetilde{u}_{0}\right)\right\|_{L^{2}(\Omega)} \leq C \varepsilon\left\|\widetilde{u}_{0}\right\|_{H^{2}\left(\mathbb{R}^{d}\right)} \leq C \varepsilon\left\|u_{0}\right\|_{H^{2}(\Omega)} .
$$


By combining (4.7) and (4.8), we have proved that

$$
\left\|p_{\varepsilon}-p_{0}-\left[\pi^{\varepsilon} S_{\varepsilon}\left(\nabla \widetilde{u}_{0}\right)-f_{\Omega} \pi^{\varepsilon} S_{\varepsilon}\left(\nabla \widetilde{u}_{0}\right)\right]\right\|_{L^{2}(\Omega)} \leq C \sqrt{\varepsilon}\left\|u_{0}\right\|_{H^{2}(\Omega)} .
$$

\section{Convergence rates for $u_{\varepsilon}$ in $L^{2}$}

To establish the sharp $O(\varepsilon)$ rate for $u_{\varepsilon}$ in $L^{2}$, we realize that

$$
\left\|u_{\varepsilon}-u_{0}\right\|_{L^{2}(\Omega)} \leq\left\|v_{\varepsilon}\right\|_{L^{2}(\Omega)}+\varepsilon\left\|\chi^{\varepsilon} S_{\varepsilon}\left(\nabla \widetilde{u}_{0}\right)\right\|_{L^{2}(\Omega)} .
$$

By using Proposition 2.6 and (3.2),

$$
\left\|\chi^{\varepsilon} S_{\varepsilon}\left(\nabla \widetilde{u}_{0}\right)\right\|_{L^{2}(\Omega)} \leq\|\chi\|_{L^{2}(Y)}\left\|\nabla \widetilde{u}_{0}\right\|_{L^{2}\left(\mathbb{R}^{d}\right)} \leq C\left\|u_{0}\right\|_{H^{2}(\Omega)} .
$$

Thus, the problem has been reduced to the proof of

$$
\left\|v_{\varepsilon}\right\|_{L^{2}(\Omega)} \leq C \varepsilon\left\|u_{0}\right\|_{H^{2}(\Omega)},
$$

for which we'd use the duality argument.

Proof of Theorem 1.1. We consider the following duality problems, for any $H \in$ $L^{2}\left(\Omega ; \mathbb{R}^{d}\right)$, let $\left(\varphi_{\varepsilon}, \sigma_{\varepsilon}\right) \in H^{1}\left(\Omega ; \mathbb{R}^{d}\right) \times L^{2}(\Omega)$ be the weak solution of the following adjoint Neumann problem of Stokes system

$$
\left\{\begin{aligned}
\mathcal{L}_{\varepsilon}^{*}\left(\varphi_{\varepsilon}\right)+\nabla \sigma_{\varepsilon} & =H-f_{\Omega} H & & \text { in } \Omega, \\
\operatorname{div}\left(\varphi_{\varepsilon}\right) & =0 & & \text { in } \Omega, \\
\left(\frac{\partial \varphi_{\varepsilon}}{\partial \nu_{\varepsilon}}\right)^{*}-\sigma_{\varepsilon} \cdot n & =0 & & \text { on } \partial \Omega,
\end{aligned}\right.
$$

and $\left(\varphi_{0}, \sigma_{0}\right) \in H^{2}\left(\Omega ; \mathbb{R}^{d}\right) \times H^{1}(\Omega)$ be the weak solution of its corresponding homogenized adjoint problem

$$
\left\{\begin{aligned}
\mathcal{L}_{0}^{*}\left(\varphi_{0}\right)+\nabla \sigma_{0} & =H-f_{\Omega} H & & \text { in } \Omega \\
\operatorname{div}\left(\varphi_{0}\right) & =0 & & \text { in } \Omega \\
\left(\frac{\partial \varphi_{0}}{\partial \nu_{0}}\right)^{*}-\sigma_{0} \cdot n & =0 & & \text { on } \partial \Omega
\end{aligned}\right.
$$

and satisfying

$$
\int_{\Omega} \varphi_{\varepsilon}=\int_{\Omega} \varphi_{0}=0, \quad \text { and } \int_{\Omega} \sigma_{\varepsilon}=\int_{\Omega} \sigma_{0}=0 .
$$


Here we have used the notation: $\mathcal{L}_{\varepsilon}^{*}=-\operatorname{div}\left(A^{*}(x / \varepsilon) \nabla\right)$ and $\mathcal{L}_{0}^{*}=-\operatorname{div}\left(\widehat{A^{*}} \nabla\right)$. We note that Theorem 1.2 continues to hold for $\mathcal{L}_{\varepsilon}^{*}$, as $A^{*}$ satisfies the same conditions as $A$. Also, by the $W^{2,2}$ estimates (2.4) for Stokes systems with constant coefficients in $C^{1,1}$ domains,

$$
\left\|\varphi_{0}\right\|_{H^{2}(\Omega)}+\left\|\sigma_{0}\right\|_{H^{1}(\Omega)} \leq C\|H\|_{L^{2}(\Omega)} .
$$

As a result, we have

$$
\left\|\varphi_{\varepsilon}-\varphi_{0}-\varepsilon \chi^{* \varepsilon} S_{\varepsilon}\left(\nabla \widetilde{\varphi}_{0}\right)\right\|_{H^{1}(\Omega)} \leq C \sqrt{\varepsilon}\left\|\varphi_{0}\right\|_{H^{2}(\Omega)} \leq C \sqrt{\varepsilon}\|H\|_{L^{2}(\Omega)} .
$$

where $\left(\chi^{*}, \pi^{*}\right)$ denotes the correctors associated with adjoint matrix $A^{*}$. Therefore through dual pairing, and integrating by parts, we obtain

$$
\begin{aligned}
\int_{\Omega} v_{\varepsilon}\left(H-f_{\Omega} H\right) & =\left\langle\mathcal{L}_{\varepsilon}^{*}\left(\varphi_{\varepsilon}\right), v_{\varepsilon}\right\rangle_{H_{0}^{-1}\left(\Omega ; \mathbb{R}^{d}\right) \times H^{1}\left(\Omega ; \mathbb{R}^{d}\right)}+\int_{\Omega} v_{\varepsilon} \nabla \sigma_{\varepsilon} \\
& =a_{\varepsilon}\left(v_{\varepsilon}, \varphi_{\varepsilon}\right)-\int_{\Omega} \sigma_{\varepsilon} \operatorname{div}\left(v_{\varepsilon}\right) .
\end{aligned}
$$

By (3.26) and (2.3), we know that

$$
\left|\int_{\Omega} \sigma_{\varepsilon} \operatorname{div}\left(v_{\varepsilon}\right)\right| \leq C\left\|\sigma_{\varepsilon}\right\|_{L^{2}(\Omega)}\left\|\operatorname{div}\left(v_{\varepsilon}\right)\right\|_{L^{2}(\Omega)} \leq C \varepsilon\|H\|_{L^{2}(\Omega)}\left\|u_{0}\right\|_{H^{2}(\Omega)} .
$$

Since $\operatorname{div}\left(\varphi_{\varepsilon}\right)=0$, then by Lemma 3.2,

$$
\begin{aligned}
\left|a_{\varepsilon}\left(v_{\varepsilon}, \varphi_{\varepsilon}\right)\right| & \leq C\left\|u_{0}\right\|_{H^{2}(\Omega)}\left[\varepsilon^{1 / 2}\left\|\nabla \varphi_{\varepsilon}\right\|_{L^{2}\left(\Omega_{2 \varepsilon}\right)}+\varepsilon\left\|\nabla \varphi_{\varepsilon}\right\|_{L^{2}(\Omega)}\right] \\
& \leq C \varepsilon\left\|u_{0}\right\|_{H^{2}(\Omega)}\|H\|_{L^{2}(\Omega)}+C \varepsilon^{1 / 2}\left\|u_{0}\right\|_{H^{2}(\Omega)}\left\|\nabla \varphi_{\varepsilon}\right\|_{L^{2}\left(\Omega_{2 \varepsilon}\right)},
\end{aligned}
$$

where we have used (2.3) for the last inequality and $C$ is independent of $\varepsilon$. By triangle inequality,

$$
\begin{aligned}
\left\|\nabla \varphi_{\varepsilon}\right\|_{L^{2}\left(\Omega_{2 \varepsilon}\right)} \leq & \left\|\nabla\left(\varphi_{\varepsilon}-\varphi_{0}-\varepsilon \chi^{* \varepsilon} S_{\varepsilon}\left(\nabla \widetilde{\varphi}_{0}\right)\right)\right\|_{L^{2}\left(\Omega_{2 \varepsilon}\right)} \\
& +\left\|\nabla \varphi_{0}\right\|_{L^{2}\left(\Omega_{2 \varepsilon}\right)}+\left\|\nabla\left(\varepsilon \chi^{* \varepsilon} S_{\varepsilon}\left(\nabla \widetilde{\varphi}_{0}\right)\right)\right\|_{L^{2}\left(\Omega_{2 \varepsilon}\right)} .
\end{aligned}
$$

Directly deriving from (15.6), we know that

$$
\left\|\nabla\left(\varphi_{\varepsilon}-\varphi_{0}-\varepsilon \chi^{* \varepsilon} S_{\varepsilon}\left(\nabla \widetilde{\varphi}_{0}\right)\right)\right\|_{L^{2}\left(\Omega_{2 \varepsilon}\right)} \leq C \sqrt{\varepsilon}\|H\|_{L^{2}(\Omega)} .
$$

By using Lemma 2.7 and (3.2) again, we get

$$
\left\|\nabla \varphi_{0}\right\|_{L^{2}\left(\Omega_{2 \varepsilon}\right)} \leq C\left(\varepsilon\left\|\nabla \varphi_{0}\right\|_{H^{1}(\Omega)}\left\|\nabla \varphi_{0}\right\|_{L^{2}(\Omega)}\right)^{1 / 2} \leq C \sqrt{\varepsilon}\left\|\varphi_{0}\right\|_{H^{2}(\Omega)} \leq C \sqrt{\varepsilon}\|H\|_{L^{2}(\Omega)} .
$$

Similarly as in (3.23), by Lemma 2.7, Proposition 2.6 and (3.2), we have

$$
\begin{aligned}
\left\|\nabla\left(\varepsilon \chi^{* \varepsilon} S_{\varepsilon}\left(\nabla \widetilde{\varphi}_{0}\right)\right)\right\|_{L^{2}\left(\Omega_{2 \varepsilon}\right)} & \leq C\left\{\left\|\left(\nabla \chi^{*}\right)^{\varepsilon} S_{\varepsilon}\left(\nabla \widetilde{\varphi}_{0}\right)\right\|_{L^{2}\left((\partial \Omega)_{2 \varepsilon}\right)}+\varepsilon\left\|\widetilde{\varphi}_{0}\right\|_{H^{2}\left(\mathbb{R}^{d}\right)}\right\} \\
& \leq C \sqrt{\varepsilon}\left\|\varphi_{0}\right\|_{H^{2}(\Omega)} \leq C \sqrt{\varepsilon}\|H\|_{L^{2}(\Omega)} .
\end{aligned}
$$


Substituting (5.11), (5.12) and (5.13) into (5.10), we have proved that

$$
\left\|\nabla \varphi_{\varepsilon}\right\|_{L^{2}\left(\Omega_{2 \varepsilon}\right)} \leq C \sqrt{\varepsilon}\|H\|_{L^{2}(\Omega)} .
$$

Therefore,

$$
\begin{aligned}
\left|a_{\varepsilon}\left(v_{\varepsilon}, \varphi_{\varepsilon}\right)\right| & \leq C \varepsilon\left\|u_{0}\right\|_{H^{2}(\Omega)}\|H\|_{L^{2}(\Omega)}+C \varepsilon^{1 / 2}\left\|u_{0}\right\|_{H^{2}(\Omega)}\left(\varepsilon^{1 / 2}\|H\|_{L^{2}(\Omega)}\right) \\
& \leq C \varepsilon\|H\|_{L^{2}(\Omega)}\left\|u_{0}\right\|_{H^{2}(\Omega)} .
\end{aligned}
$$

Hence, by using (5.15) and (5.8), we already proved that for any $H \in L^{2}\left(\Omega ; \mathbb{R}^{d}\right)$

$$
\begin{aligned}
\left|\int_{\Omega} v_{\varepsilon}\left(H-f_{\Omega} H\right)\right| & \leq\left|a_{\varepsilon}\left(v_{\varepsilon}, \varphi_{\varepsilon}\right)\right|+\left|\int_{\Omega} \sigma_{\varepsilon} \operatorname{div}\left(\varphi_{\varepsilon}\right)\right| \\
& \leq C \varepsilon\|H\|_{L^{2}(\Omega)}\left\|u_{0}\right\|_{H^{2}(\Omega)} .
\end{aligned}
$$

Therefore, since $\int_{\Omega} u_{\varepsilon}=\int_{\Omega} u_{0}=0$,

$$
\begin{aligned}
\left|\int_{\Omega} v_{\varepsilon} H\right| & \leq\left|\int_{\Omega} v_{\varepsilon}\left(H-f_{\Omega} H\right)\right|+\left|\left(f_{\Omega} H\right) \int_{\Omega} v_{\varepsilon}\right| \\
& \leq C \varepsilon\|H\|_{L^{2}(\Omega)}\left\|u_{0}\right\|_{H^{2}(\Omega)}+C\left|\left(f_{\Omega} H\right) \int_{\Omega} \varepsilon \chi^{\varepsilon} S_{\varepsilon}\left(\nabla \widetilde{u}_{0}\right)\right| \\
& \leq C \varepsilon\|H\|_{L^{2}(\Omega)}\left\|u_{0}\right\|_{H^{2}(\Omega)},
\end{aligned}
$$

where we have used (5.16) for the second inequality and Proposition 2.6 for the last, for any $H \in L^{2}\left(\Omega ; \mathbb{R}^{d}\right)$. By duality, this implies

$$
\left\|v_{\varepsilon}\right\|_{L^{2}(\Omega)} \leq C \varepsilon\left\|u_{0}\right\|_{H^{2}(\Omega)} .
$$

where $C$ is independent of $\varepsilon$. Therefore we have completed the proof.

Acknowledgment The author wants to thank the anonymous referees for their very helpful comments and suggestions.

\section{References}

[1] M. Avellaneda and F. Lin, Compactness methods in the theory of homogenization, Comm. Pure Appl. Math. 40 (1987), no. 6, 803-847.

[2] D. Cioranescu, A. Damlamian, and G. Griso, Periodic unfolding and homogenization, C. R. Math. Acad. Sci. Paris 335 (2002), no. 1, 99-104.

[3] _ The periodic unfolding method in homogenization, SIAM J. Math. Anal. 40 (2008), no. 4, 1585-1620.

[4] J. Geng and Z. Shen, Convergence Rates in Parabolic Homogenization with TimeDependent Periodic Coefficients, arXiv:1604.06735 (2016). 
[5] M. Giaquinta and G. Modica, Nonlinear systems of the type of the stationary NavierStokes system, J. Reine Angew. Math. 330 (1982), 173-214.

[6] G. Griso, Error estimate and unfolding for periodic homogenization, Asymptot. Anal. 40 (2004), no. 3-4, 269-286.

[7] _ Interior error estimate for periodic homogenization, Anal. Appl. (Singap.) 4 (2006), no. 1, 61-79.

[8] S. Gu, Convergence rates in homogenization of Stokes systems, J. Differential Equations 260 (2016), no. 7, 5796-5815.

[9] _ Homogenization of Stokes Systems with Periodic Coefficients, (2016), Theses and Dissertations-Mathematics. Paper 39.

[10] S. Gu and Z. Shen, Homogenization of Stokes systems and uniform regularity estimates, SIAM J. Math. Anal. 47 (2015), no. 5, 4025-4057.

[11] V. V. Jikov, S. M. Kozlov, and O. A. Oleynik, Homogenization of Differential Operators and Integral Functionals, Springer-Verlag, Berlin, 1994.

[12] C. E. Kenig, F. Lin, and Z. Shen, Convergence rates in $L^{2}$ for elliptic homogenization problems, Arch. Ration. Mech. Anal. 203 (2012), no. 3, 1009-1036 (English).

[13] _ Periodic homogenization of Green and Neumann functions, Comm. Pure Appl. Math. 67 (2014), no. 8, 1219-1262.

[14] C. E. Kenig and Z. Shen, Homogenization of elliptic boundary value problems in Lipschitz domains, Math. Ann. 350 (2011), no. 4, 867-917 (English).

[15] L Layer potential methods for elliptic homogenization problems, Comm. Pure Appl. Math. 64 (2011), no. 1, 1-44.

[16] J.-L. Lions, Asymptotic expansions in perforated media with a periodic structure, Rocky Mountain J. Math. 10 (1980), no. 1, 125-140. MR 573867

[17] S. Moskow and M. Vogelius, First-order corrections to the homogenised eigenvalues of a periodic composite medium. A convergence proof., Proc. Roy. Soc. Edinburgh Sect. A 127 (1997), no. 6, 12631299.

[18] D. Onofrei and B. Vernescu, Error estimates for periodic homogenization with nonsmooth coefficients, Asymptot. Anal. 54 (2007), no. 1-2, 103-123.

[19] M. A. Pakhnin and T. A. Suslina, Homogenization of the elliptic Dirichlet problem: error estimates in the $\left(L^{2} \rightarrow H^{1}\right)$-norm, Funct. Anal. Appl. 46 (2012), no. 2, 155159. 
[20] - Operator error estimates for the homogenization of the elliptic Dirichlet problem in a bounded domain, St. Petersburg Math. J. 24 (2013), no. 6, 949-976.

[21] S. E. Pastukhova, On some estimates from the homogenization of problems in plasticity theory. (Russian), Dokl. Akad. Nauk 406 (2006), no. 5, 604-608.

[22] Z. Shen, Boundary estimates in elliptic homogenization, arXiv:1505.00694 (2015).

[23] Z. Shen and J. Zhuge, Convergence rates in periodic homogenization of systems of elasticity, to appear in Proc. Amer. Math. Soc. (2015).

[24] T. A. Suslina, Homogenization of the Dirichlet problem for elliptic systems: $L_{2}$ operator error estimates, Mathematika 59 (2013), no. 2, 463-476.

[25] _ Homogenization of the Neumann problem for elliptic systems with periodic coefficients, SIAM J. Math. Anal. 45 (2013), no. 6, 3453-3493.

[26] R. Temam, Navier-Stokes Equations. Theory and Numerical Analysis., Studies in Mathematics and its Applications, vol. 2, North-Holland Publishing Co., AmsterdamNew York-Oxford, 1977.

[27] V. V. Zhikov and S. E. Pastukhova, On operator estimates for some problems in homogenization theory, Russ. J. Math. Phys. 12 (2005), no. 4, 515-524.

Shu Gu, Department of Mathematics, The Florida State University, Tallahassee, FL 32306, USA

E-mail: gu@math.fsu.edu; gushu0329@uky.edu

September 20, 2018 